\author{
A. Adamkiewicz, A. Waliszyn \\ Maritime University of Szczecin, Faculty of Marine Engineering, Waty Chrobrego 1-2, \\ 70-500 Szczecin, Poland \\ a.adamkiewicz@am.szczecin.pl
}

\title{
STUDIES OF EROSION RESISTANCE OF PROTECTIVE COATS ON THE SURFACES OF MACHINE ELEMENTS WASHED WITH FLUIDS
}

\begin{abstract}
This article indicated at erosion as one of the causes of degradation of surfaces washed by fluids and conditions of its occurrence. Corrosive - erosive theory of metal surface degradation has been discussed linking it with an instance of destructive processes taking place in cylinder liner blocks of combustion engines. Physics and conditions influencing processes on liquid - washed operational surface phase boundary have been justified. Out of the contemporary hypotheses explaining the physics of cavitation erosion, the bubble theory has been considered. A mathematical model of erosion has been presented in the context of cavitation implosion energy determining crash interactions of liquid cumulative fluxes on the washed surface. Occurring plastic deformations have been graphically explained linking them with the occurrence of fatigue micro-cracks and later with erosive pits. Influence of initial steel hardness on intensity of cavitation erosion has been checked. Discussion of ways to increase metal surface resistance to cavitation erosion has been carried out.
\end{abstract}

Keywords: cavitation, erosion, Reynolds number, damping protective coats, corrosion - cavitation degradation

\section{INTRODUCTION}

Corrosive - erosive damage of fluid washed surfaces of hydraulic and thermal machines intensify degradation of their technical condition, at the same time lowering durability of the washed elements. The cause of erosion occurrence is most often due to cavitation in the operating areas of machine or facility $[1,2,3]$.

At present, the corrosive-erosive theory of metal surface damage has been acclaimed. Practically all specialists think that the pre- cause of damage to fluid washed surfaces is the turbulent interaction of liquid with a metal surface layer resulting from explosions of cavitation bubbles. For example, erosive damage to liners and combustion engine cylinder blocks, which is manifested with concentration of deep pits, takes place as a result of complex cooperation of mechanical and electrochemical processes damaging metals, cavitation erosion and electrochemical corrosion. As a result of piston strikes when it passes the top dead centre (TDC) and bottom dead centre (BDC), the cylinder liner vibrates with high frequency, which leads to a change of cooling liquid flux speed on the surfaces of liners and cylinder blocks. Locally occurring depression and pressure increase favour disturbances in flux continuity. That generates plenty of cavitation bubbles filled with vapour, gas or their mixture in areas with lowered pressure $[4,5,6]$. 
Conditions of cavity occurrence are highly influenced by changes in the values of speed and pressure of the liquid flow. Therefore, it is reasonable to consider all conditions of interactions on the processes taking place at the liquid-operational surface border of the facility.

Change of pressure in a chosen point of a metal element surface washed by liquid can be evaluated throughout a non-dimensional coefficient of local depression of liquid pressure [7]

$$
\xi=\left(\frac{\vartheta_{0}}{\vartheta_{i}}\right)^{2}-1
$$

where: $\vartheta_{0}$ - mean speed of the liquid washing the surface of the element;

$v_{i}$ - flux speed in a chosen point $i$ on the surface of the element.

The largest depression $n_{i}$ will be in the place where the value of $\xi$ coefficient is maximal

where: $p_{0}-$ mean pressure of the liquid ;

$$
p_{i}=p_{0}-q \xi
$$

$q$-pressure increase speed of the liquid flux $\quad q=p v_{i}^{2} / 2$

$\rho-$ liquid density.

The process of cavitation starts when pressure $p i$ reaches the value of saturated vapour of the liquid $p_{N}$ at the temperature of the environment. Formation of cavitation bubbles takes place in the flux washing the metal surface in the tapering area, where the liquid flux increases its speed. Implosion takes place in areas of lowered speed, where the flux faces resistance or flows through widening canals $[4,5,6]$.

Corrosive -erosive damage of hydraulic machine surfaces washed with fluids and cooled surfaces of thermal machines causes significant degradation of their technical condition and decreases their durability. Erosion is most frequently due to liquid cavitation in the operational areas of hydraulic/flow machines and equipment. Despite a significant number of studies on the cavitation phenomenon and occurrence of erosive damage on metal surfaces still there is an open issue of the physics of interaction of the liquid flow on the surfaces of protective coats in particular the dumping ones $[6,8,9]$. Those issues comprise the following:

1. The influence of flow canal shape changes on the conditions of cavitation occurrence;

2. Dependence of criteria numbers of cavitation beginning, $K_{k}$, and critical parameters of the beginning of cavitation erosion $D_{\text {ke }}$ on the Reynolds number, $\mathrm{Rn}$, at different levels of external dynamic interaction of the liquid washing the surfaces;

3. Relationships between $D_{k e}$ and $K_{k}$ for different levels of similarity.

Conditions of cavity occurrence are highly influenced by changes in the values of speed and pressure of the liquid flow. Therefore, it is reasonable to consider all conditions of interactions on the processes taking place at the liquid-operational surface border of the facility.

\section{MATHEMATICAL MODEL OF EROSIVE CAVITATION}

For the needs of solving those issues, processes taking place in the near-wall liquid layer adjacent to the canal wall were treated as a vortex area where there is a distribution of horizontal liquid velocity components (along the $\mathrm{X}_{1}$ axis) with the vortex element at the $\mathrm{X}_{3}$ distance from the bordering surface. The equation of state of the vortex area is similar to the differential equation of micro vortex diffusion in the following form: 


$$
\frac{\partial \Delta \vartheta_{1}}{\partial t}+\vartheta_{3} \frac{\partial \Delta \vartheta_{1}}{\partial x_{3}}=\frac{\partial^{2} \Delta \vartheta_{1}}{\partial x_{2}^{2}}
$$

where: $\Delta v_{1}$ is the change of a lengthwise velocity component $\quad{ }_{1}(\mathrm{x}, \mathrm{t})[5,10,11]$. Fig 1.

The distribution of velocity vectors in the fluid washing the vortex element is shown in

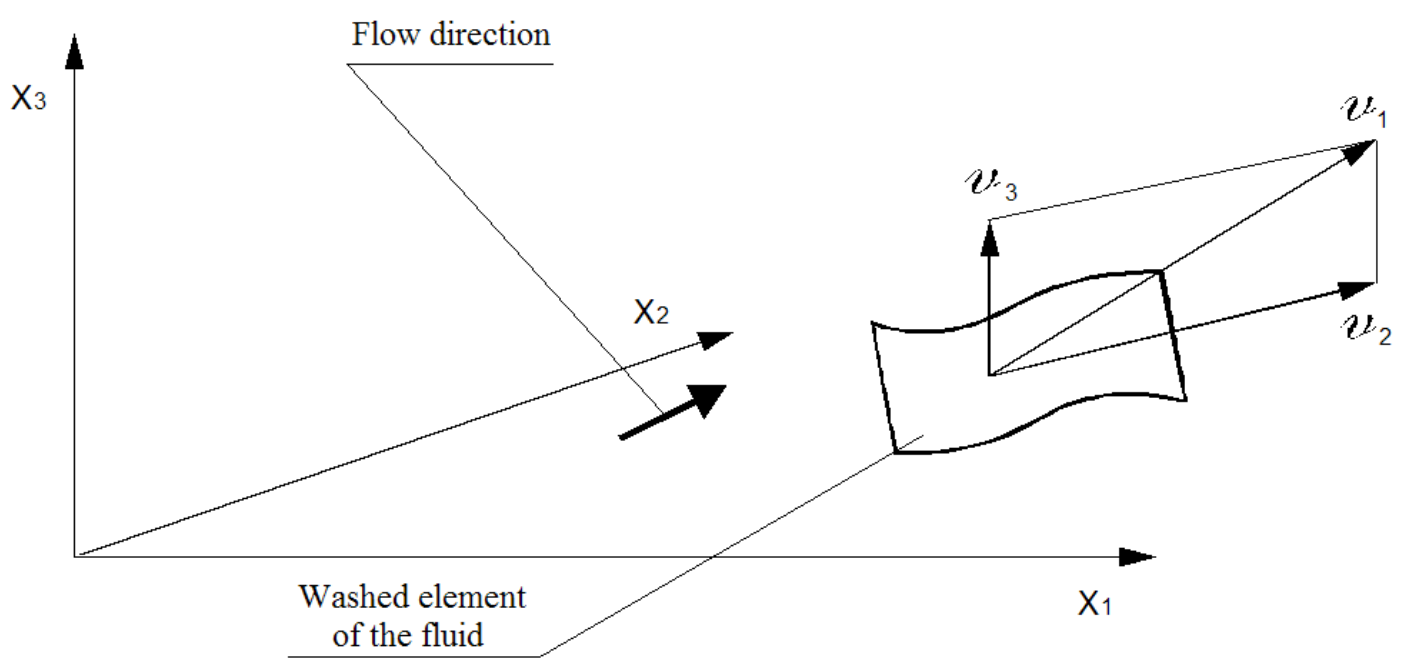

Fig. 1. Distribution of vectors of velocity in the fluid washing a rotation element

In the case when protective dumping coats are used, knowledge of the influence of their deformations due to the interaction of the flow with the vertical component of the liquid velocity is necessary. With turbulent flow along the surface with a protective dumping coat equation (3) will become $[1,2,9]$ :

$$
\frac{\partial \Delta \vartheta_{1}}{\partial t}+\left(\vartheta_{3}-\Delta \vartheta_{3}\right) \frac{\partial \Delta \vartheta_{1}}{\partial x_{3}}=\frac{\partial^{2} \Delta \vartheta_{1}}{\partial x_{3}^{2}}
$$

where:

$\Delta \vartheta_{3}$ - is the decrease of $\vartheta_{3}(x, t)$ velocity as a measurement of flux displacing the deformed dumping co $t$ - time.

Pressure $p\left(x_{3}, t\right)$ of the liquid influences the $\vartheta_{3}(x, t)$ velocity according to the following relation:

$$
\vartheta_{3}(x, l) \equiv-\frac{x_{R}}{p} \frac{\partial p}{\partial t}
$$

then for the dumping surface, equation (3) becomes:

$$
\vartheta_{3}-\Delta \vartheta_{3}=\frac{\left(x_{\mathrm{I}}-\Delta x_{2}\right)}{p} \frac{\partial p}{\partial t}
$$

where:

$x_{3}$ - may be considered as local plastic deformation of the dumping surface. 
Deformation of the dumping surface $\left(\mathrm{x}_{3}\right)$ and appearance of a vertical component of the liquid velocity $\vartheta_{3}(x, t)$ makes the flow laminar decreasing the probability of cavitation occurrence. As a result of turbulence appearing in the near-wall layer of the liquid, the following equation can be formulated for the new variables:

$$
\theta(x, t)=1-\frac{2}{\sqrt{\pi}} \int_{0}^{\xi} \exp \left(z^{2} d z\right)
$$

where:

$$
\xi=\left(\mathrm{x}_{3}-\Delta x_{3}\right) / h(t), \quad \text { and } \quad h(t)=\frac{2}{p}\left(\int p^{2} d t\right)^{\frac{2}{2}}
$$

$\mathrm{h}$ - non-dimensional coefficient.

In the case when the surface is washed by a liquid without deformations $\left(\mathrm{x}_{3}=0\right)$, the function becomes:

$$
\Delta \theta\left(x_{3}=0, t\right)=\frac{2}{\sqrt{\pi}} \int_{0}^{z\left(-\Delta x_{3}\right)} \exp \left(-z^{2}\right) d z \neq 0
$$

Currently, the physics of cavitation erosion is explained by such basic hypotheses as $[6,11]$

1. Hydromechanical interactions of micro flows of liquids and shock waves;

2. Thermodynamic interactions resulting from the occurrence of high temperatures at the implosion of cavitation bubbles;

3. Sudden chemical reactions basing on the assumption that at the moment when cavitation bubble implodes, gases within it and liquids around it decompose;

4. Electrochemical cavitation interactions based on chemical and electrochemical processes taking place in metal crystals.

The most widespread theory of cavitation erosion occurrence becomes the bubble theory, according to which damage to metal takes place as a result of a turbulent liquid interaction with significant pressure and temperature at the moment of vapour-gas bubble implosion.

\section{CAVITATION IMPLOSION ENERGY}

During an implosion of vapour-gas bubble, liquid flows of great speed, up to $34 \mathrm{~m} / \mathrm{s}$, appear at the boundary of its surface. At the same time the pressure of the liquid at the boundary of the bubble implosion with the metal surface washed by water may exceed $\left(510^{6}\right)$ $\mathrm{Pa}$. Then the striking energy of particles of the cumulative flux is equal:

where:

$$
E-\int w v_{k}^{2}\left[\frac{\eta_{1} \omega}{6 k T}+\frac{\eta_{n}}{c^{2} p}\right]^{-1},
$$

$m$ - mass of the liquid in the striking flux,

$\mathrm{v}$ - speed of the cumulative flux, 
$\eta_{1} i \eta_{2}-$ viscosity coefficient of the liquid before and after the bubble implosion

$\omega$ - internal friction coefficient of the molecules of the liquid,

$\mathrm{k}$ - Boltzmann constant,

$T$ - temperature,

$\mathrm{c}$ - sound speed,

$f$ - coefficient accounting for the influence of bubble collision

$\rho$ - fluid density.

Striking interaction of cumulative liquid fluxes on the surface of the washed metal leads to its plastic deformation of the surface and an increase of its hardness - strain. In the reinforced upper layers of the metal which are still under further striking interactions of cumulative liquid fluxes, as a result of fatigue, micro cracks appear and their further development leads to the occurrence of erosion pits in the form of craters. Figure 2 shows a diagram of a metal damaged as a result of cumulative flux interaction $[6,12,13]$.

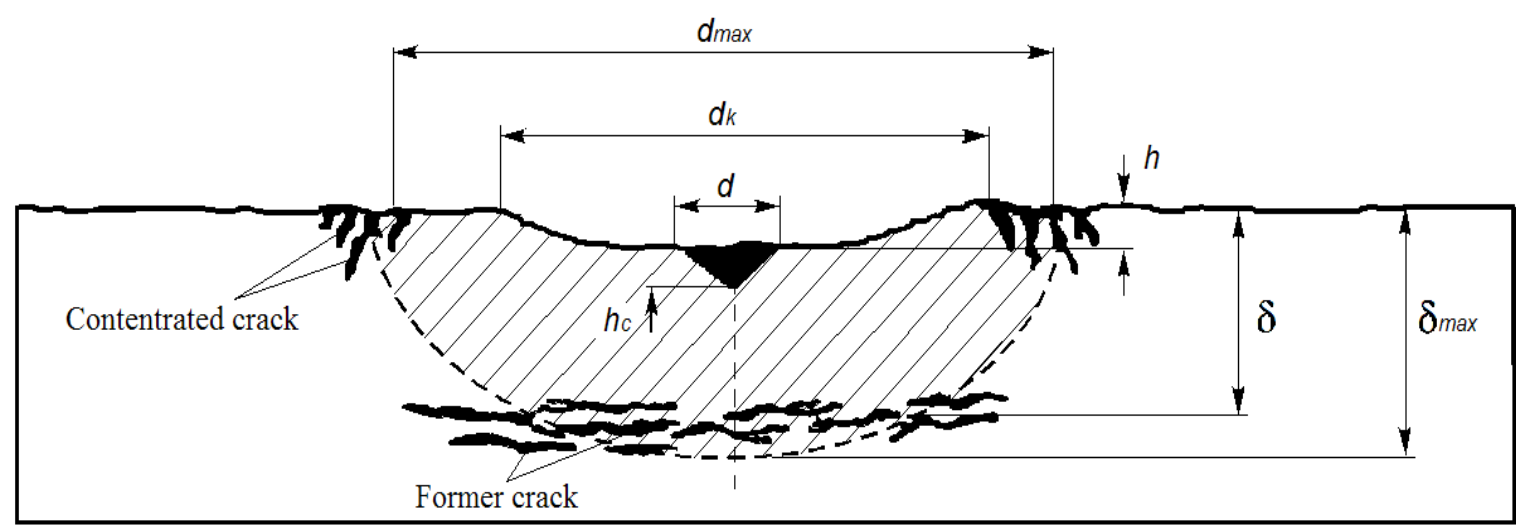

Fig. 2. A diagram of metal damage resulting from erosion cavitation $d$ max - maximum diameter corresponding to the area of concentrated cracks $\mathrm{dk}$ pit diameter, d diameter of oxidation product occurrence area, ; $\delta$ depth of metal damage

Damage to the metal takes place after reaching critical values of load which is characteristic for fatigue damage of materials. Frequently, not very effective methods of increasing hardness of surfaces that are affected by cavitation erosion are suggested in technical literature in order to avoid erosion damage. However, in paper [12] intensity of cavitation erosion is described by the following relation

$$
I=\operatorname{const} \cdot H^{n}
$$

where : $\mathrm{J}$ - erosion intensity ( $\mathrm{mg} / \mathrm{mm}^{2}$ hour);

$\mathrm{H}-$ metal surface hardness $(\mathrm{HB})$; 
$\mathrm{n}$ - exponent with values ranging from 2,78 (for carbon steels) to 0 (for chromium alloy steels). The influence of initial steel hardness on the intensity of cavitation erosion is shown in Fig. 3 [7].

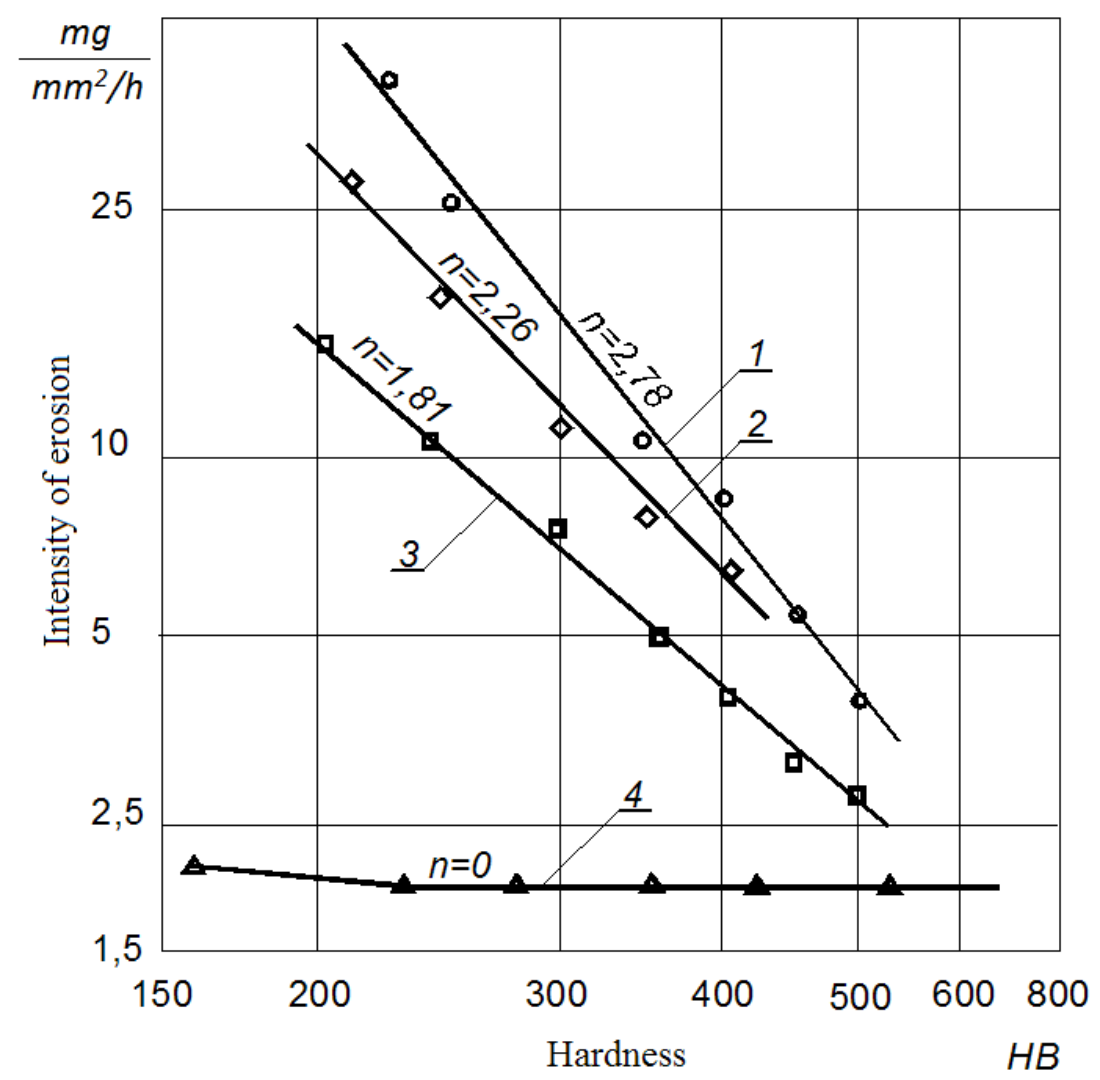

Fig. 3. Intensity of cavitation erosion in relation to initial steel hardness 1- S40 i S1Cr40; Cr5V3; 2 - 1Cr13, 2Cr13, Mn20Si1; 3 - 1Cr18Ni3Mn4Cu2; 4 - 30Cr10Mn10

An analysis of results shown in the graph shows that the hardness of the upper surface of the metal is not the only value determining metal resistance to erosive damage.

\section{CONCLUSION}

Erosive damage to alloy steel with lower hardness in comparison to that of steels used in the experiment was much smaller $[13,14,15]$. It shows that in order to increase the resistance to cavitation erosion the surface of the metal must be more plastic.

The increase of resistance to cavitation erosion of metal surfaces can be reached throughout:

- making the working liquid flow more laminar;

- covering metal surfaces with protective coats;

- dumping vibrations of parts/elements in the case of vibration cavitation.

Liquid flows can be laminar thanks to hydraulic system organisation into such where speeds of liquid flows do not exceed critical values corresponding to limiting values of Reynolds criterion. The critical value, determining the beginning of cavitation is given by the following mathematical form [7]: 


$$
K_{k}=\frac{k_{*}^{2}}{c_{l}^{v}}\left(C_{j}-\Delta C_{j}^{*}\right)
$$

where:

$K_{k}^{0}$ i $C_{j}^{0}$ - cavitation number and resistance coefficient of the near-wall layer of the non- laminar liquid flow;

$j$-meter of operations

$\Delta C_{j}^{*}$ - depending on the structure of the liquid flow is described by (10):

$$
\Delta C_{j}^{*}=1,33\left(1-\frac{1}{F}\right) / \sqrt{R e}
$$

where :

$e^{F}$ - limiting plasticity of the metal surface washed by the liquid [7].

An analysis of expressions (9-10) for the resistance coefficient $C_{j}^{*}$ indicates that with decreasing the criterion Reynolds number and increasing plasticity of the washed surface, the resistance of the upper layer increases. Respectively, cavitation number $\mathrm{K}_{\mathrm{k}}$, characterizing the conditions of the beginnings of cavitation phenomena and damage connected with them, decreases.

\section{REFERENCES}

1. Bolewski Ł., Szkodo M., Kmieć M., Cavitation erosion degradation of Belzona ${ }^{\circledR}$ coatings, Advances in Materials Science, 17(1) (2017), DOI: 10.1515/adms-2017-0002.

2. Krella A., Cavitation degradation model of hard thin PVD coatings, Advances in Materials Science, 10(3) (2010) 27-36, DOI: 10.2478/v10077-010-0010-4.

3. Amann T., Waidele M., Kailer A., Analysis of mechanical and chemical mechanisms on cavitation erosioncorrosion of steels in salt water using electrochemical methods, Tribology International, 124 (2018) 238-246, DOI: 10.1016/j. triboint.2018.04.012

4. Waliszyn A.: Methods to avoid surface damage of metal construction due to corrosion in engine cooling systems. [In] Implications in Production Processes. Institute of Examinations and Scientific Studies, Gorzów Wlkp., (2016) 68-80 (in Polish).

5. Valishin A. G., Matviejevskij O. O.: Modeling cavitation - erosive stability of dumping materials and coats. Problems of machine construction and reliability of machines, No 3, Moskva, (2008), 43-50 (in Russian).

6. Kwok C. T., Man H. C., Cheng F. T.: Cavitation erosion and damage mechanisms of alloys with duplex structures. Materials Science and Engineering, A242 (1998) 108-120.

7. Steller J., Krella A., Koronowicz J., Janicki W.: Towards quantitative assessment of material resistance to cavitation erosion. Wear, 258 (2005) 604-613. 
8. Heathcock C.J., Protheroe B.E., Ball A.: Cavitation erosion of stainless steels. Wear, 81(2) (1982) 311-327.

9. Kim J H, Lee M H., A Study on Cavitation Erosion and Corrosion Behavior of Al-, Zn-, Cu-, and Fe-Based Coatings Prepared by Arc Spraying, Journal Of Thermal Spray Technology, 19 (6) (2010) 1224-1230, DOI: 10.1007/s11666-010-9521-0.

10. Yang D., Yu A., Ji B., Zhou, J., Luo X., Numerical analyses of ventilated cavitation over a 2-D NACA0015 hydrofoil using two turbulence modeling methods, Journal Of Hydrodynamics, 30(2) (2018) 345-356, DOI: 10.1007/s42241-018-0032-7.

11. Krumenacker, L., Fortes-Patella, R., Archer, A., Numerical estimation of cavitation intensity, IOP Conference Series-Earth and Environmental Science, 22 (2014) Article Number: UNSP 052014, DOI: $10.1088 / 1755-1315 / 22 / 5 / 052014$.

12. Pogodaev L.I., Kuzmin A. A.: Erosion of materials in ship technology in uniform liquid and gasous environments. Monograph, Sankt-Petersburg, (2004) 379 (in Russian).

13. Ciubotariu, CR., Secosan E., Marginean, G., Frunzaverde D., Campian V. C., Experimental study regarding the cavitation and corrosion resistance of stellite 6 and self-fluxing remelted coatings, Strojniski Vestnik-Journal of Mechanical Engineering, 62 (3) (2016) 154-162, DOI: 10.5545/svjme.2015.2663.

14. Kumar, H., Chittosiya, C., Shukla, V.N., HVOF sprayed WC based cermet coating for mitigation of cavitation, erosion \& abrasion in hydro turbine blade, Materials Today-Proceedings, 5 (2) (2018) 6413-6420.

15. Yu A., Luo X., Ji B., Analysis of ventilated cavitation around a cylinder vehicle with nature cavitation using a new simulation method, Science Bulletin, 60(21) (2015) 1833-1839, DOI: $10.1007 / \mathrm{s} 11434-015-0916-7$. 